\title{
Primary gastrointestinal stromal tumor of the liver in an anorectal melanoma survivor: A case report
}

\author{
YUNG-YEH SU ${ }^{1}$, NAI-JUNG CHIANG ${ }^{2,3}$, CHUN-CHIEH WU ${ }^{4}$ and LI-TZONG CHEN ${ }^{1-3}$ \\ ${ }^{1}$ Department of Internal Medicine, Kaohsiung Medical University Hospital, Kaohsiung Medical University, Kaohsiung 807; \\ ${ }^{2}$ National Institute of Cancer Research, National Health Research Institutes, Tainan 704; ${ }^{3}$ Division of Hematology/Oncology, \\ Department of Internal Medicine, National Cheng Kung University Hospital, Tainan 701; ${ }^{4}$ Department of Pathology, \\ Kaohsiung Medical University Hospital, Kaohsiung Medical University, Kaohsiung 807, Taiwan, R.O.C.
}

Received May 17, 2014; Accepted February 10, 2015

DOI: $10.3892 / \mathrm{ol} .2015 .3561$

\begin{abstract}
The majority of gastrointestinal stromal tumors (GISTs) arise in the stomach and small intestine; primary GIST of the liver is extremely rare. GISTs share specific immunohistological features with melanoma, therefore, determining a definitive diagnosis can be difficult. However, electron microscopy can be used to aid the differential diagnosis of GIST. The present study reports the first case of a KIT/platelet-derived growth factor receptor $\alpha$ (PDGFRA) wild-type, primary GIST arising from the liver in a long-term survivor of anorectal melanoma. The patient underwent APR for treatment of malignant melanoma of the anorectum in 2001 with no adjuvant therapy and remained disease-free until 2009. In 2009, the patient presented with a solitary, rapidly growing hypervascular liver tumor, which was subsequently diagnosed as a primary GIST of the liver. Imatinib treatment (400 mg/day) was initially administered for two months, however, disease progression occurred. Therefore, the patient underwent chemotherapy with doxorubicin $\left(50 \mathrm{mg} / \mathrm{m}^{2}\right)$ and cisplatin $\left(50 \mathrm{mg} / \mathrm{m}^{2}\right)$ every three weeks. Although this temporarily resulted in stable disease, progression occurred five months later. Finally, oral sunitinib (37.5 mg/day) was administered; however, the patient succumbed to the disease one month later. Despite the current GIST patient exhibiting a poor response to imatinib, the present study highlights the importance of considering a second primary malignancy and performing immunohistochemical analysis upon the occurrence of a newly developed lesions in long-term remission cancer survivors.
\end{abstract}

Correspondence to: Dr Yung-Yeh Su, Department of Internal Medicine, Kaohsiung Medical University Hospital, Kaohsiung Medical University, 100 Ziyou 1st Road, Sanmin, Kaohsiung 807, Taiwan, R.O.C.

E-mail: awakengod@hotmail.com

Key words: primary gastrointestinal stromal tumor of the liver, anorectal melanoma, imatinib, wild-type tumor

\section{Introduction}

Anorectal melanoma, the third most common site of mucosal melanoma, is a rare tumor with an incidence rate of 1.7 cases per million in the global population (1). Surgery, including abdominoperineal resection (APR) or wide local excision (WLE), is the primary treatment strategy for localized disease; however, these two surgical approaches result in similarly poor clinical outcomes, with five-year recurrence rates of 79 and $81 \%$ following APR and WLE, respectively (2). Furthermore, the five-year overall survival rate following curative resection ranges from 15 to $22 \%$, with the majority of patients succumbing to recurrent disease. Recurrence predominantly occurs in the liver, followed by the lung and brain $(3,4)$.

Gastrointestinal stromal tumors (GISTs), characterized by the expression of cluster of differentiation (CD) 117 and a high incidence of $K I T$ or platelet-derived growth factor receptor $\alpha$ (PDGFRA) mutations, are uncommon tumors with an incidence range of 6-15 cases per million individuals (5). The majority of GISTs arise in the stomach and small intestine, and GISTs originating from the esophagus, colon and extra-gastrointestinal organs, such as the retroperitoneum and liver, are rare (6). To the best of our knowledge, only five cases of primary hepatic GIST have previously been reported in the English literature (7-11). The most common KIT mutation sites involve exon $11(66.1 \%)$ followed by exon 9 (13\%); however, $12 \%$ of cases exhibit no detectable KIT mutation and are therefore categorized as KIT wild-type GISTs (12).

The present study reports the case of a long-term survivor of anorectal melanoma who, seven years after APR surgery, developed a solitary rapidly growing tumor in the liver.

\section{Case report}

A 58-year-old Taiwanese male was diagnosed with malignant melanoma of the anorectum and underwent APR at Kaohsiung Medical University Hospital (Kaosiung, Taiwan) in 2001. Microscopically, the anorectal tumor was composed of spindle-shaped neoplastic cells beneath the squamous epithelium with brownish pigments and adjacent junctional activity. Furthermore, the tumor was immunohistochemically positive for human melanoma black (HMB-45) and S-100 (Fig. 2A-D). 
No surgical margin or lymph node involvement was apparent, therefore, stage I anorectal melanoma was diagnosed. The patient did not receive any adjuvant therapy and was regularly followed up by physical examination every two weeks at Kaohsiung Medical University Hospital for five years after surgery.

In 2009, after a further two years and at the age of 65 years, the patient presented to the Department of General Surgery at Kaohsiung Medical University Hospital with a two-month history of general malaise, loss of appetite and progressively worsening dull pain over the epigastrium. An appropriate physical examination revealed no abnormalities, with the exception of the APR surgical scar on the abdominal wall. However, a follow-up contrast-enhanced computed tomography (CT) scan revealed a $12 \times 11-\mathrm{cm}$ solitary tumor within the left lobe of the liver (Fig. 1A). Serum $\alpha$-fetoprotein, carcinoembryonic antigen and carbohydrate antigen 19-9 levels were all within normal limits. Subsequently, a percutaneous CT-guided biopsy of the liver tumor was performed. Microscopically, the liver tumor was composed of spindle cells arranged in interlacing fascicles with hyperchromatic, round or oval to spindle pleomorphic nuclei. Immunohistochemical (IHC) staining revealed that the neoplastic cells were diffusely and strongly positive for CD117, but negative for HMB-45, S-100 melan-A and CD34. Furthermore, the mitotic activity of the tumor cells was five mitoses per 10 high-power fields. Retrospectively, positive CD117 staining was also observed in the anorectal melanoma and the basal layer squamous epithelium. The results of the histological and IHC staining of the anorectal melanoma are shown in Fig. 2A-D, and the liver tumor in Fig. 2F-I. In addition, polymerase chain reaction followed by direct sequencing was performed using DNA extracted from formalin-fixed, paraffin-embedded (FFPE) tissue, revealing that the anorectal melanoma and hepatic tumor were negative for $B R A F^{V 600 E}$ (data not shown).

To confirm the diagnosis, transmission electron microscopy (TEM) of the FFPE-derived tumors was performed to determine the ultrastructure of the two tumors. Analysis of the anorectal tumor showed a number of electron-dense granules around the nucleus, which were considered to be atypical melanosomes, and inconspicuous microtubules within the cisternal space of the cytoplasm (Fig. 2E). These features were considered to correspond with the diagnosis of melanoma. Due to damage of the fine structures of the small paraffin-embedded tissue during formalin fixation and subsequent xylene treatment, artifacts were present in the sample and a collapsed cytoplasmic structure was observed. Thus, the quality of the liver TEM image was poor. Therefore, no villous cytoplasmic processes or dispersed intermediate filaments, which are considered to be characteristic ultrastructure features of GISTs, could be identified (13). However, hyperchromatic nuclei and a vesicular cytoplasm without melanosomes were present (Fig. 2J), indicating that the liver tumor may have a different histology from the prior melanoma.

Despite testing negative for KIT and PDGFRA mutations, the liver tumor was diagnosed as primary GIST of the liver based on its IHC profiling (diffusely strongly CD117-positive, and negative for HMB-45 and S-100), and TEM features when compared with anorectal melanoma, as well as the lack of other intra-abdominal tumors identified on abdominal CT imaging. Due to the presence of left portal vein invasion, the

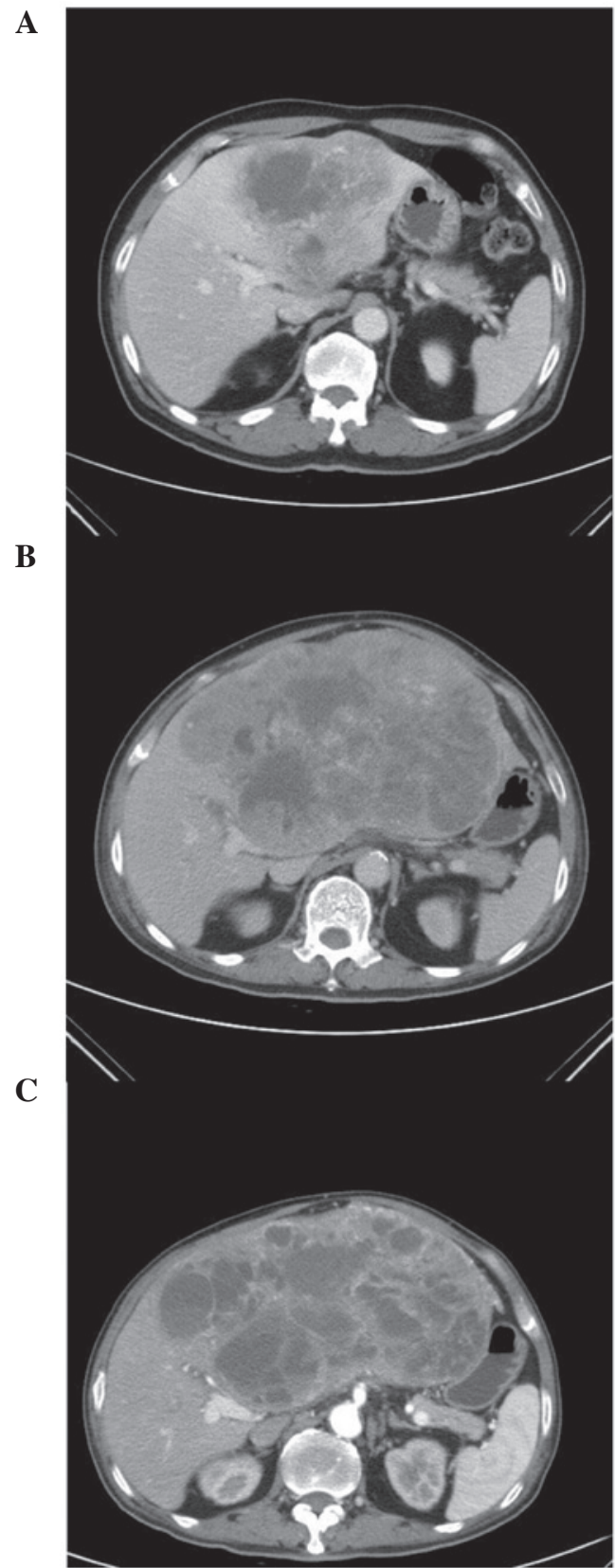

Figure 1. (A) Initial CT scan indicating a heterogeneous contrast-enhanced mass in the left hepatic lobe with left portal vein invasion at diagnosis. (B) CT scans three months after imatinib treatment (dose, $400 \mathrm{mg} /$ day), and (C) two months after chemotherapy with doxorubicin (dose, $50 \mathrm{mg} / \mathrm{m}^{2}$ ) and cisplatin (dose, $50 \mathrm{mg} / \mathrm{m}^{2}$ ). CT, computed tomography.

hepatic GIST was considered as unresectable, therefore, oral imatinib was administered at a dose of $400 \mathrm{mg} /$ day for two months. However, a follow-up CT scan after two months of imatinib therapy demonstrated that the tumor had progressed to $22 \times 13 \mathrm{~cm}$ in size (Fig. 1B). While awaiting the approval of sunitinib from regulatory authorities, systemic chemotherapy consisting of $50 \mathrm{mg} / \mathrm{m}^{2}$ doxorubicin and $50 \mathrm{mg} / \mathrm{m}^{2}$ cisplatin was administered every three weeks for five months. This regimen resulted in symptomatic improvement manifesting as a decrease in dull epigastric pain and tumor size by palpation after the first cycle of treatment. Thus, this chemotherapeutic treatment strategy was continued. A follow-up CT scan, conducted two months later, demonstrated that the tumor had stabilized 


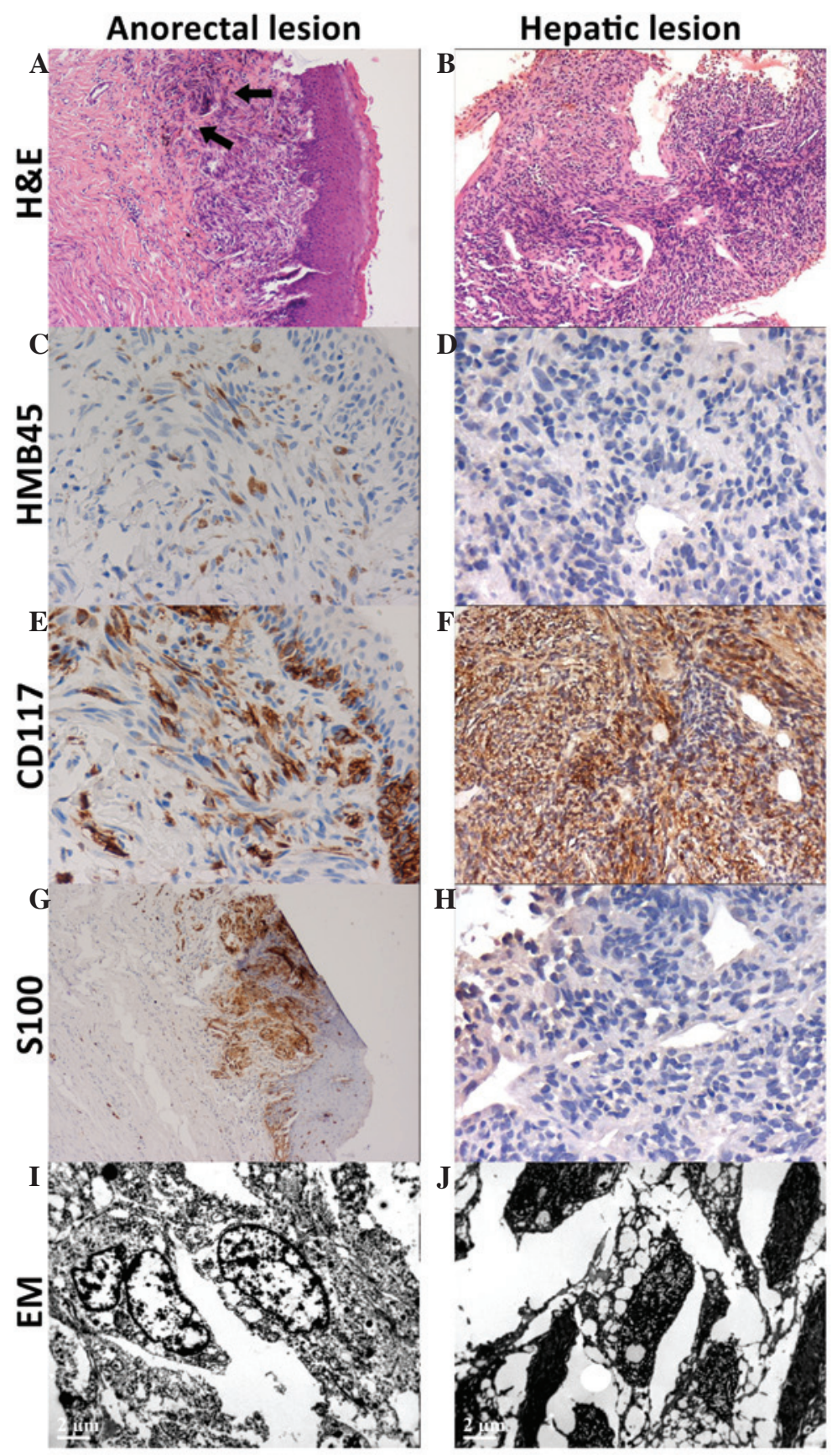

Figure 2. Histology: (A-H) IHC analysis and (I and J) EM of the primary anorectal melanoma and second primary hepatic gastrointestinal stromal tumors. For the anorectal lesion, (A) H\&E staining reveled subepithelial spindle neoplastic cells with brownish pigments (arrows), which were positive for (C) HMB45, (E) CD117 and (G) S-100 in IHC analysis, and (I) positive for atypical melanosome in EM examination. For the hepatic lesion, (B) H\&E staining revealed spindle neoplastic cells arranged in whorls or intervening fascicles, which were (D) negative for HMB45, (F) strongly positive for CD117 and $(\mathrm{H})$ negative for S-100 in IHC staining. (J) EM showed hyperchromatic nuclei and vesicular cytoplasm. IHC, immunohistochemical; H\&E, hematoxylin and eosin; HMB-45, human melanoma black-45; CD117, cluster of differentiation 117; EM, electronic microscopy.

with minimal regression to a size of 19x11 cm (Fig. 1C). The chemotherapy was continued for an additional three months, substituting with $37.5 \mathrm{mg} /$ day sunitinib after disease progression. However, the patient did not respond to sunitinib and succumbed to the disease one month later.

\section{Discussion}

The current study describes the case of a unique patient who was found to have a solitary hypervascular liver tumor that was subsequently diagnosed as a second primary GIST of the liver by IHC. The patient had undergone APR for a stage I anorectal melanoma seven years prior. Clinically, it is not possible to distinguish between a metastatic melanoma and a metastatic or primary GIST in the liver, as the two types of tumor can be hypervascular in the early arterial phase of a CT scan, and grow rapidly. Considering the history of anorectal melanoma and the absence of a KIT/PDGFRA mutation during genotyping, the diagnosis of GIST in the present patient may be disputable. However, if the patient did not have 
a history of mucosal melanoma, the tumor could be easily diagnosed as GIST based on the results of the CD117 staining, irrespective of its KIT/PDGFRA genotyping. Therefore, a final diagnosis of hepatic GIST was determined, based on the diffusely strong CD117 staining, negativity for HMB-45 and S100 (two positive markers noted in his anorectal melanoma) and the difference in ultrastructure between the two tumors, as noted by performing TEM.

Initially, the diagnosis of GIST was considered to be more favorable for the current patient compared with those exhibiting metastatic melanoma, with disease-specific median survival periods of 19.0 and 13.3 months, respectively (14). However, following the approval of imatinib in 2002, results indicated that metastatic or unresectable GIST was more treatable compared with metastatic melanoma, with a notable increase in survival to 38.4-60 months (according to the kinase genotype) (15). Imatinib markedly improved the survival of GIST patients, regardless of the KIT/PDGFR mutation status. Despite imatinib typically producing a poorer response in wild-type GIST compared with exon 11-mutated GIST, imatinib does provide a benefit to patients with wild-type GIST, exhibiting a disease control rate of $>60 \%$ (15). Furthermore, in patients with wild-type GIST, Asian populations appear more likely to benefit from imatinib treatment compared with Western populations. In the CALGB 150105 study, clinical benefit rates were 78-95\% in the Asian population and 64\% in the Western population (15). Additionally, five-year survival rates of 60-70\% and $40-45 \%$ were noted for Asian and Western populations, respectively (15-17). Although the differences may have been caused by the small-size bias of Asian studies, the possibility of higher pharmacokinetic parameters in Asian populations following fixed-dose imatinib treatment should also be considered. Despite the increased benefit in Asian populations, the current patient did not receive any benefit from imatinib treatment and the tumor progressed rapidly within two months, which indicates that the nature of primary hepatic GIST may be different from those arising in the alimentary tract.

Tothe best of our knowledge, to date, only five cases of primary hepatic GIST have been previously reported (Table I) (7-11). Among these five cases, two cases received imatinib therapy $(8,9)$. One of the imatinib-treated cases lacked mutation analysis data, however, the patient exhibited a good response to imatinib, resulting in a disease-free status for three years (8). The other imatinib-treated case had no mutation of KIT exon 11 (only KIT exon 11 was analyzed) and responded well to imatinib, with a long-term survival period of nine years (9). As only a small number of cases of primary hepatic GIST have been reported thus far, the correlation between the KIT genotype and response to imatinib, a tyrosine kinase inhibitor, remains inconclusive; thus, additional studies are required to address the issue.

In conclusion, despite the poor response to imatinib of the primary hepatic KIT/PDGFRA wild-type GIST in the current patient, the present study highlights the importance of considering a second primary malignancy and performing immunohistochemical analysis upon the occurrence of a newly developed lesions in long-term remission cancer survivors. With the emergence of modern molecular technologies, such as next-generation sequencing, it may be possible to precisely determine genetic alterations that exist between the two tumors. This may aid in determining the nature of the second 
tumor (delayed recurrence versus second primary), as well as providing useful information to guide therapeutic treatment of the disease.

\section{References}

1. Weinstock MA: Epidemiology and prognosis of anorectal melanoma. Gastroenterology 104: 174-178, 1993.

2. Yeh JJ, Shia J, Hwu WJ, et al: The role of abdominoperineal resection as surgical therapy for anorectal melanoma. Ann Surg 244: 1012-1017, 2006

3. Homsi J and Garrett C: Melanoma of the anal canal: a case series. Dis Colon Rectum 50: 1004-1010, 2007.

4. Aytac B, Adim SB, Yerci O and Yilmazlar T: Anorectal malignant melanomas: experience of Uludag University. Kaohsiung J Med Sci 26: 658-662, 2010.

5. Ho MY and Blanke CD: Gastrointestinal stromal tumors: disease and treatment update. Gastroenterology 140: 1372-1376.e2, 2011

6. Reith JD, Goldblum JR, Lyles RH and Weiss SW: Extragastrointestinal (soft tissue) stromal tumors: an analysis of 48 cases with emphasis on histologic predictors of outcome. Mod Pathol 13: 577-585, 2000.

7. Hu X, Forster J and Damjanov I: Primary malignant gastrointestinal stromal tumor of the liver. Arch Pathol Lab Med 127: 1606-1608, 2003.

8. De Chiara A, De Rosa V, Lastoria S, et al: Primary gastrointestinal stromal tumor of the liver with lung metastases successfully treated with STI-571 (imatinib mesylate). Front Biosci 11: 498-501, 2006.
9. Ochiai T, Sonoyama T, Kikuchi S, et al: Primary large gastrointestinal stromal tumor of the liver: report of a case. Surg Today 39: 633-636, 2009.

10. Luo XL, Liu D, Yang JJ, Zheng MW, Zhang J and Zhou XD: Primary gastrointestinal stromal tumor of the liver: a case report. World J Gastroenterol 15: 3704-3707, 2009.

11. Yamamoto H, Miyamoto Y, Nishihara Y, et al: Primary gastrointestinal stromal tumor of the liver with PDGFRA gene mutation. Hum Pathol 41: 605-609, 2010.

12. Corless CL, Fletcher JA and Heinrich MC: Biology of gastrointestinal stromal tumors. J Clin Oncol 22: 3813-3825, 2004.

13. Park SH, Kim MK, Kim H, et al: Ultrastructural studies of gastrointestinal stromal tumors. J Korean Med Sci 19: 234-244, 2004

14. Balch CM, Buzaid AC, Soong SJ, et al: Final version of the American Joint Committee on Cancer staging system for cutaneous melanoma. J Clin Oncol 19: 3635-3648, 2001.

15. Heinrich MC, Owzar K, Corless CL, et al: Correlation of kinase genotype and clinical outcome in the North American Intergroup Phase III Trial of imatinib mesylate for treatment of advanced gastrointestinal stromal tumor: CALGB 150105 Study by Cancer and Leukemia Group B and Southwest Oncology Group. J Clin Oncol 26: 5360-5367, 2008.

16. Yeh CN, Chen TW, Lee HL, et al: Kinase mutations and imatinib mesylate response for 64 Taiwanese with advanced GIST: preliminary experience from Chang Gung Memorial Hospital. Ann Surg Oncol 14: 1123-1128, 2007.

17. Kim TW, Ryu MH, Lee H, et al: Kinase mutations and efficacy of imatinib in Korean patients with advanced gastrointestinal stromal tumors. Oncologist 14: 540-547, 2009. 